\title{
Excited-state spin coherence of a single nitrogen-vacancy centre in diamond
}

\author{
G. D. Fuchs ${ }^{1}$, V. V. Dobrovitski ${ }^{2}$, D. M. Toyli ${ }^{1}$, F. J. Heremans ${ }^{1}$, C. D. Weis ${ }^{3}$, T. Schenkel ${ }^{3}$ \\ and D. D. Awschalom ${ }^{1 \star}$
}

\begin{abstract}
Nitrogen-vacancy centres in diamond are a solid-state analogue of trapped atoms, with fine structure in both the ground and excited states that may be used for advanced quantum control. These centres are promising candidates for spin-based quantum information processing ${ }^{1-3}$ and magnetometry ${ }^{4-6}$ at room temperature. Knowledge of the excited-state (ES) structure and coherence is critical to evaluating the ES as a room-temperature quantum resource, for example for a fast, optically gated swap operation with a nuclear-spin memory ${ }^{7}$. Here we report experiments that probe the ES-spin coherence of single nitrogen-vacancy centres. Using a combination of pulsed-laser excitation and nanosecond-scale microwave manipulation, we observed ES Rabi oscillations, and multipulse resonant control enabled us to study coherent ES electron/nuclear-spin interactions. To understand these processes, we developed a finite-temperature theory of ES spin dynamics that also provides a pathway towards engineering longer ES spin coherence.
\end{abstract}

Intense experimental effort to understand the ES levels of nitrogen-vacancy (NV) centres has focused on spectroscopic investigations both at room temperature ${ }^{7-9}$ and at low temperature ${ }^{9-11}$. The short ES orbital lifetime (10-20 ns), however, presents a challenge for the measurement of ES spin coherence. The recent development of subnanosecond spin manipulation of individual $\mathrm{NV}$ centres ${ }^{12}$ puts these investigations within reach, with the fundamental goal of understanding ES-spin dynamics and decoherence mechanisms. Through these experiments, we establish the presence of room-temperature ES-spin coherence despite rapid phononmediated transitions between orbital branches ${ }^{13}$ that might be expected to destroy the quantum phase of the spin.

$\mathrm{NV}$ centres are a naturally occurring lattice defect in diamond. They consist of a substitutional nitrogen atom and an adjacent carbon vacancy. The ground state (GS) is a spin-1 orbital singlet $\left({ }^{3} \mathrm{~A}\right)$, whereas the ES is a spin-1 orbital doublet $\left({ }^{3} \mathrm{E}\right)$. All ES spin levels (spin quantum number $m_{\mathrm{s}}=0, \pm 1$ ) exhibit spontaneous decay by photon emission. Further radiationless relaxation from the ES $m_{\mathrm{s}}= \pm 1$ levels through an intersystem crossing causes the $\mathrm{NV}$ photoluminescence intensity $\left(I_{\mathrm{PL}}\right)$ to vary linearly with the $m_{\mathrm{s}}=0$ spin population and enables optical pumping into the $m_{\mathrm{s}}=0$ spin $\operatorname{state}^{14}$ (Fig. 1a).

Before probing ES spin dynamics we first characterize the orbital relaxation. Figure $1 \mathrm{~b}$ shows the decay of $I_{\mathrm{PL}}$ as a function of time for a single NV centre at room temperature excited using a pulsed laser (Fig. 1c). The slowest decay rate (purple) occurs when the NV centre is optically polarized into $m_{\mathrm{s}}=0$ with no further spin manipulation. The fastest decay rate (blue) occurs when a $1 \mathrm{~ns}$ microwave $\pi$ pulse on the ES $m_{\mathrm{s}}=0$ to -1 transition is applied simultaneously with optical excitation. Microwave $\pi$ pulses at later delays ( 7 and $15 \mathrm{~ns}$ ) abruptly change the initialized state decay rate to the $m_{\mathrm{s}}=-1$ decay rate (green and gold, respectively) as seen previously ${ }^{8}$.

The average number of photons emitted by an NV centre during the collection window $T$ evolves as

$$
N(T)=p_{0}\left(1-\mathrm{e}^{-\gamma_{0} T}\right)+\left(1-p_{0}\right)\left(1-\mathrm{e}^{-\gamma_{1} T}\right) \gamma_{0} / \gamma_{1}
$$

where $p_{0}$ is the initial polarization of the NV centre in the $m_{\mathrm{s}}=0$ state, $\gamma_{0}$ is the decay rate from the $m_{\mathrm{s}}=0$ state and $\gamma_{1}$ is the decay rate from the $m_{\mathrm{s}}= \pm 1$ state including the intersystem relaxation. After a $\pi$ pulse is applied at time $\tau_{\pi}<T$, the populations of $m_{\mathrm{s}}=0$ and $m_{\mathrm{s}}= \pm 1$ interchange, and the evolution follows

$$
\begin{aligned}
N(T)= & p_{0}\left(1-\mathrm{e}^{-\gamma_{0} \tau_{\pi}}\right)+\left(1-p_{0}\right)\left(1-\mathrm{e}^{-\gamma_{1} \tau_{\pi}}\right) \gamma_{0} / \gamma_{1} \\
& +p_{0} \mathrm{e}^{-\gamma_{0} \tau_{\pi}}\left(1-\mathrm{e}^{-\gamma_{1}\left(T-\tau_{\pi}\right)}\right) \gamma_{0} / \gamma_{1} \\
& +\left(1-p_{0}\right) \mathrm{e}^{-\gamma_{1} \tau_{\pi}}\left(1-\mathrm{e}^{-\gamma_{0}\left(T-\tau_{\pi}\right)}\right)
\end{aligned}
$$

where we have ignored the $\pi$-pulse duration. To determine $\gamma_{0}, \gamma_{1}$ and $p_{0}$, we integrate the experimental data in Fig. $1 \mathrm{~d}$ and fit to equations (1) and (2) (Fig. 1d, inset). We found $\gamma_{0}=0.0563 \pm 0.0043 \mathrm{~ns}^{-1}, \gamma_{1}=0.111 \pm 0.014 \mathrm{~ns}^{-1}$ and $p_{0}=0.84 \pm 0.08$. The corresponding lifetimes $\left(\tau_{i}=1 / \gamma_{i}\right)$ are $\tau_{0}=17.8 \pm 1.4 \mathrm{~ns}$ and $\tau_{-1}=9.0 \pm 1.2 \mathrm{~ns}$. It is necessary to take $p_{0}$ into account to obtain good simultaneous fits and to obtain correct results for the experiments described below. Details are available in the Supplementary Information.

To probe the ES spin coherence, we measure ES Rabi oscillations by setting the microwave pulse delay to zero and varying the pulse duration. Figure 2a shows ES Rabi measurements at magnetic field $B=1,276 \mathrm{G}$ and four values of microwave power. The ES Rabi frequencies are consistent with GS Rabi oscillations measured using the same microwave power at $B=261 \mathrm{G}$, where the Larmor frequency $\omega_{0} / 2 \pi$ for the $m_{\mathrm{s}}=0$ to -1 transition is also $2.14 \mathrm{GHz}$. It is noteworthy that the ES Rabi oscillations decay faster than $\gamma=\left(\gamma_{0}+\gamma_{1}\right) / 2=0.084 \pm 0.008 \mathrm{~ns}^{-1}$ (lifetime of $11.9 \pm 1.1 \mathrm{~ns}$ ), the rate expected purely from spontaneous decay to the GS.

The enhanced ES spin decoherence rate motivates a deeper theoretical investigation of the ES spin dynamics. The right-hand panel of Fig. 2b shows the ES level structure $e^{9-11,14}$ where $m_{s}$ is a good quantum number at large magnetic field. The ES orbital doublet is split by strain into two branches. The energy splitting for a given spin transition is unique to each branch, with a transition frequency $\omega_{0 \pm} / 2 \pi$, where \pm denotes the high-/low-energy orbital branch. At room temperature there are stochastic, phonon-mediated transitions between the two orbital branches at rate $R$ (ref. 13).

${ }^{1}$ Center for Spintronics and Quantum Computation, University of California, Santa Barbara, California 93106, USA, ${ }^{2}$ Ames Laboratory and lowa State University, Ames, lowa 50011, USA, ${ }^{3}$ Lawrence Berkeley National Laboratory, Berkeley, California 94720, USA. *e-mail: awsch@physics.ucsb.edu. 
a

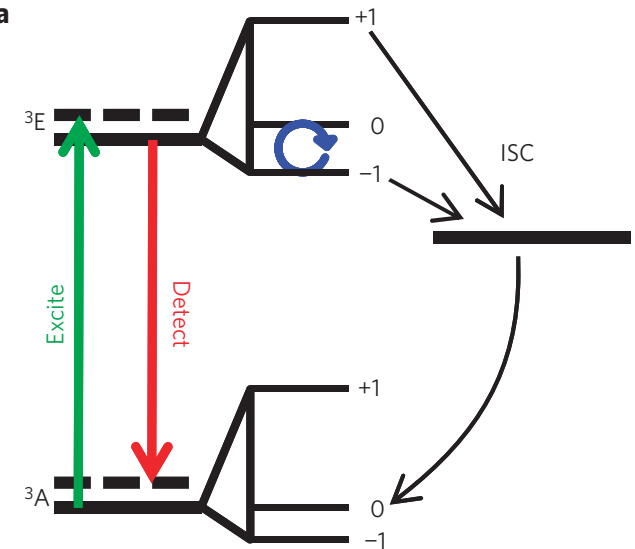

c

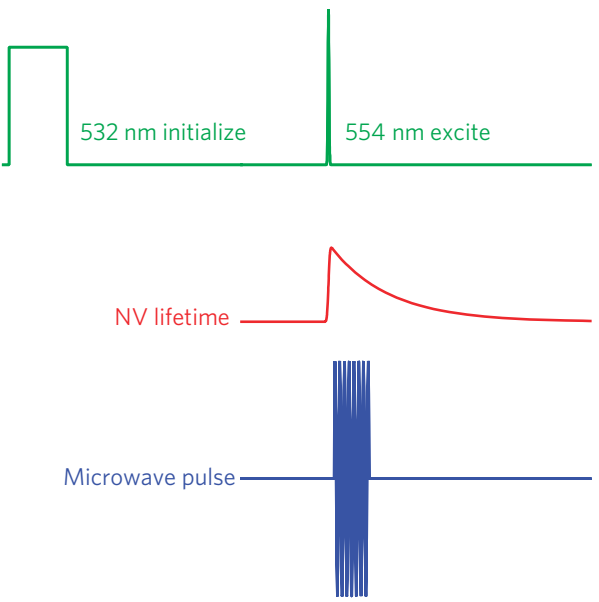

b

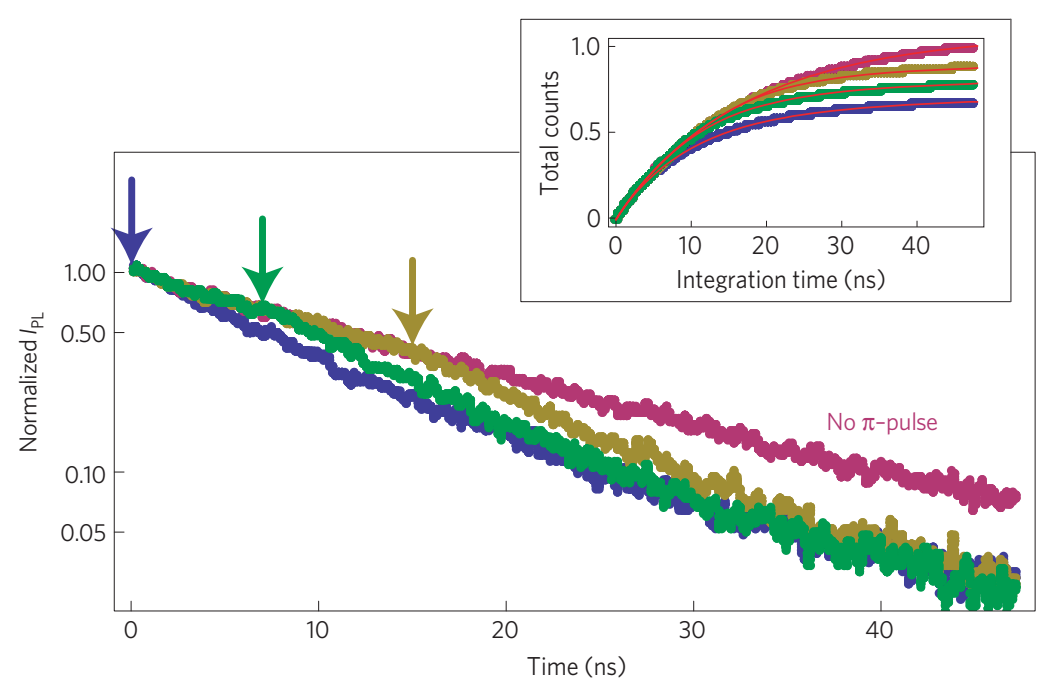

Figure 1 | Optical relaxation of NV centres. $\mathbf{a}$, Simplified level diagram of NV centres. $\mathbf{b}$, Photoluminescence as a function of time after the optical pulse. The purple data are for no microwave pulse, and the blue, green and gold data are for a microwave $\pi$ pulse applied on the ES transition at 0,7 and 15 ns delays after the optical pulse, respectively. Arrows indicate the microwave pulse timing. Inset: Integrated curves with the same colour labels overlaid with a fit to equations (1) and (2) as described in the text. c, Timing diagram.

Although these orbital transitions are spin conserving at large magnetic field, they cause motional narrowing: the two sets of ES spin resonances merge into a single set ${ }^{9,11,15}$. Each transition appears at the average frequency $\omega_{0}=\left(\omega_{0+}+\omega_{0-}\right) / 2$. In the rotating frame of this average resonance frequency, occupation of the \pm orbital branch corresponds to a longitudinal effective magnetic field $\pm B_{0}= \pm \hbar\left(\omega_{0+}-\omega_{0-}\right) /\left(2 g_{\text {es }} \mu_{\mathrm{B}}\right)$, where $\hbar$ is Planck's constant, $\mu_{\mathrm{B}}$ is the Bohr magneton and $g_{\mathrm{es}}$ is the ES $g$ factor. Phonon-mediated orbital transitions cause the effective field to switch between $+B_{0}$ and $-B_{0}$, producing spin dephasing at a rate $\Gamma=B_{0}^{2} / 2 R$. Depending on the spin transition, the external magnetic field and the strain on the NV centre, $B_{0}$ may be as large as $5.5 \mathrm{GHz}(2 \mathrm{kG})$. According to recent observations, $R$ is in the range of $1 \mathrm{THz}$ (ref. 13).

Following the approach of refs 16 and 17 we derive the equations of motion for the density matrix of the spin under motional narrowing, intense Rabi driving and spontaneous orbital decay. To compare with the experiment, we calculate the average number of photons emitted within the measurement window as a function of the microwave pulse duration. We fit each Rabi curve in Fig. 2a to the theory (solid lines). Full details of the theory and fits are described in the Supplementary Information. From these fits we extract the total ES spin coherence time $\tau^{*}$, which includes both the spontaneous orbital decay $(\gamma)$ and spin dephasing $(\Gamma)$. The values we obtain from fitting the data are plotted in the inset of Fig. 2a along with the spin-dependent orbital lifetimes. As expected, $\tau^{*}$ is independent of the microwave driving field $H_{1}$. The average of $\tau^{*}$ from all four fits gives $\tau^{*}=5.8 \pm 0.9 \mathrm{~ns}$ (red dashed line in Fig. 2b). The corresponding spin-dephasing time is $1 / \Gamma=10.9 \pm 3.3 \mathrm{~ns}$, which is comparable to the lifetime from orbital relaxation $1 / \gamma=11.9 \pm 1.1 \mathrm{~ns}$, indicating that both processes are important. This is in contrast to situations where orbital relaxation may be ignored, such as nuclear magnetic resonance measurements of rare-earth-ion ensembles, where the ES orbital lifetimes are much longer than the nuclear-spin coherence ${ }^{18}$.

Although we analyse the data using the full theory we also present simplified expressions that, although unsuitable for quantitative analysis, clarify the basic physical behaviour. To first order in the small quantity $\delta=\left(\gamma_{0}-\gamma_{1}\right) / 2$, Rabi dynamics are described by $I_{\mathrm{PL}}(t)=A-B \cos (\Omega t+\phi) \exp [-(\gamma+\Gamma / 2) t]$. Here $t$ is time, $\Omega=\sqrt{H_{1}^{2}-\Gamma^{2} / 4}$ is the renormalized Rabi frequency and $A, B$ and $\phi$ are constants. The total decay rate is a sum of the orbital decay rate $\gamma$ and spin dephasing at a rate $\Gamma / 2$. The factor of $1 / 2$ appears because the dephasing from a longitudinal field affects only the transverse spin component. 
a

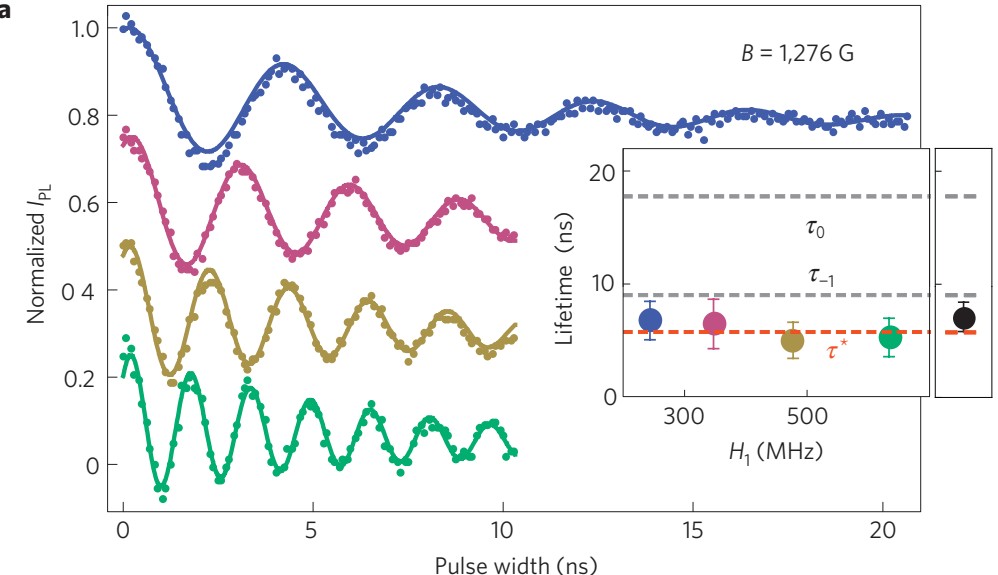

b

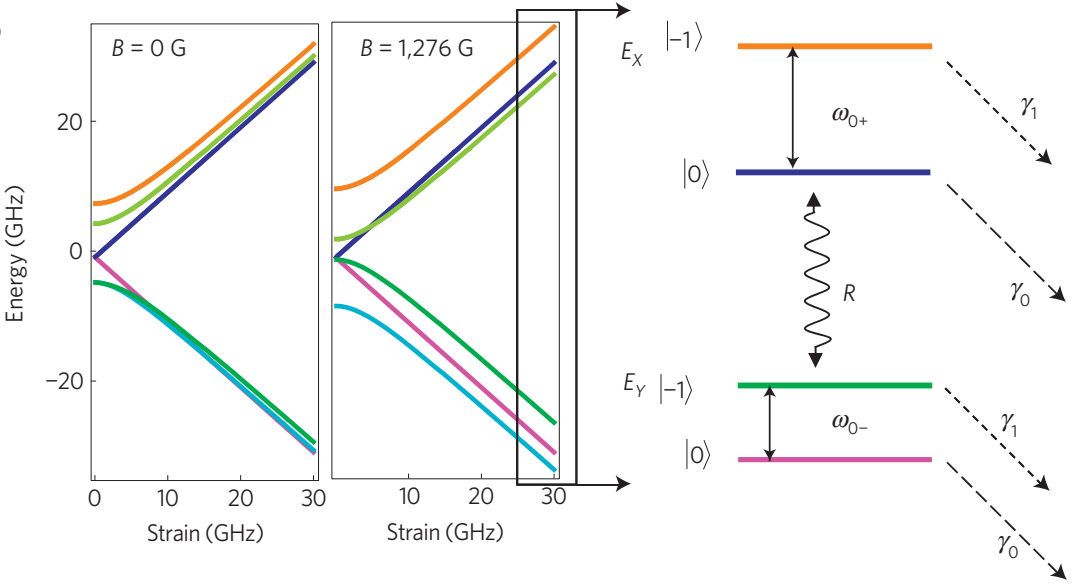

Figure 2 | ES spin dynamics of a single NV centre. a, ES Rabi oscillations for four microwave driving powers at $B=1,276 \mathrm{G}$. The Rabi frequencies from top to bottom are $244 \pm 5,348 \pm 7,477 \pm 8$ and $634 \pm 7 \mathrm{MHz}$. Inset, left panel: $\tau^{*}$ determined from fits to each Rabi curve plotted as a function of driving field $H_{1}$. The red dashed line is the average of all four points weighted by their uncertainties. For comparison, the orbital lifetimes for each spin state are also shown (grey dashed lines). Inset, right panel: $\tau^{*}$ from a fit to the Hahn-echo measurements, plotted on the same scale for comparison with Rabi measurements. $\mathbf{b}$, ES levels as a function of strain at $B=0 \mathrm{G}$ and $B=1,276 \mathrm{G}$. For scale, $1 \mathrm{GHz}$ strain corresponds to roughly $1 \mathrm{MPa}$ (ref. 11 ). For a given value of strain, there are rapid phonon-mediated transitions between the $E_{X}$ and $E_{Y}$ orbital branches at rate $R$. Within each branch, there is a Larmor frequency $\omega_{0 \pm} / 2 \pi$. There is also a spin-dependent decay rate $\gamma_{0}, \gamma_{1}$ from each spin sub-level into the orbital GS. The phonon-mediated transitions induce motional narrowing, causing the two separate resonances to appear as one resonance at the average frequency.

To investigate coherent interactions between the ES electronic spin and the ${ }^{15} \mathrm{~N}$ nuclear spin, we also carried out a two-pulse Ramsey experiment that is illustrated in Fig. 3a with a Bloch sphere representation. The spin is initialized in the $m_{\mathrm{s}}=0$ state along $+Z$. Immediately after optical excitation, we apply a microwave $\pi / 2$ pulse to bring the spin into a superposition state along $+Y$. This state is sensitive to all longitudinal fields, including the dephasing field $B_{0}$ and the $61 \mathrm{MHz}$ ES hyperfine interaction ${ }^{7}$. At the end of a free evolution period $\tau$, we apply a second $\pi / 2$ pulse to project the spin along $-Z$ for measurement. In the simplified theory, we obtain $I_{\mathrm{PL}}(t)=A_{1}+B_{1} \cos \left(A_{\mathrm{HF}} t\right) \exp [-(\Gamma+\gamma) t]$, where $A_{1}$ and $B_{1}$ are constants and $A_{\mathrm{HF}}$ is the ES hyperfine coupling. Note that, as the spin has no longitudinal component during free evolution, $\Gamma$ is not reduced. The results of our measurements are plotted in Fig. $3 \mathrm{~b}$ along with two predictions from the full theory using $\tau^{*}=5.8 \mathrm{~ns}$. The first (solid line) includes the hyperfine interaction and the second (dashed line) does not. Even on this short timescale, the ES hyperfine coupling has a strong influence on the coherent spin dynamics.

As the hyperfine interaction is coherent, it may be reversed by inserting a $\pi$ pulse in the centre of the free-evolution period, as in the three-pulse Hahn-echo sequence outlined in Fig. 4a. The $\pi$ pulse does not reverse spin dephasing due to orbital telegraphing, which occurs on timescales much shorter than the free evolution time. Thus, the simplified theoretical expression for the evolution is $I_{\mathrm{PL}}(t)=A_{2}+B_{2} \exp [-(\Gamma+\gamma) t]$, and $A_{\mathrm{HF}}$ does not appear. The data are plotted in Fig. $4 \mathrm{~b}$ along with a fit (solid line) to the full theory for this pulse sequence. We obtain $\tau^{*}=7.1 \pm 1.3 \mathrm{~ns}$, which agrees with the Rabi measurements of $\tau^{*}$ within the uncertainty (Fig. 2a, inset: right-hand panel).

The strong ES hyperfine interaction may be a promising route to an optically controlled swap gate between the NV electronic spin and its intrinsic nitrogen nuclear spin. The nitrogen nuclear spin is attractive as a quantum memory because they are present in every $\mathrm{NV}$ centre with the same coupling strength and because nuclear spins have long coherence times ${ }^{3}$. The ES hyperfine coupling has been used to polarize the ${ }^{15} \mathrm{~N}$ nuclear spin of the NV centre and to increase the spin-dependent contrast of $I_{\mathrm{PL}}$ (refs 7,19-21). Our studies demonstrate that controlled coherent interactions between the nuclear spin and the ES electronic spin are also possible. One scheme is outlined in Fig. $4 \mathrm{~d}$. Working near the avoided crossing of the motionally narrowed ES levels $(B \sim 500 \mathrm{G})$ where the eigenstates of the coupled electron-nuclear spin states become hybridized, the NV centre can be optically pumped into the $|0,+1 / 2\rangle$ state in the $\left|m_{s}, m_{I}\right\rangle$ basis ${ }^{19,22}$. Next, a GS microwave $\pi$ pulse rotates the NV electronic state into $|-1,+1 / 2\rangle$, followed by a green 


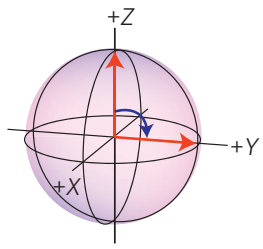

$\pi / 2$

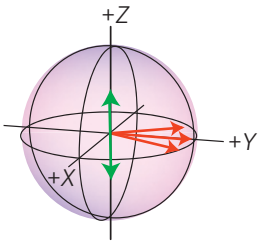

Free evolution

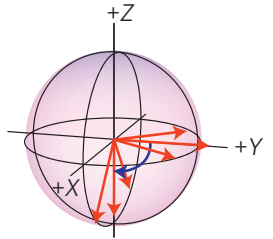

$\pi / 2$

b

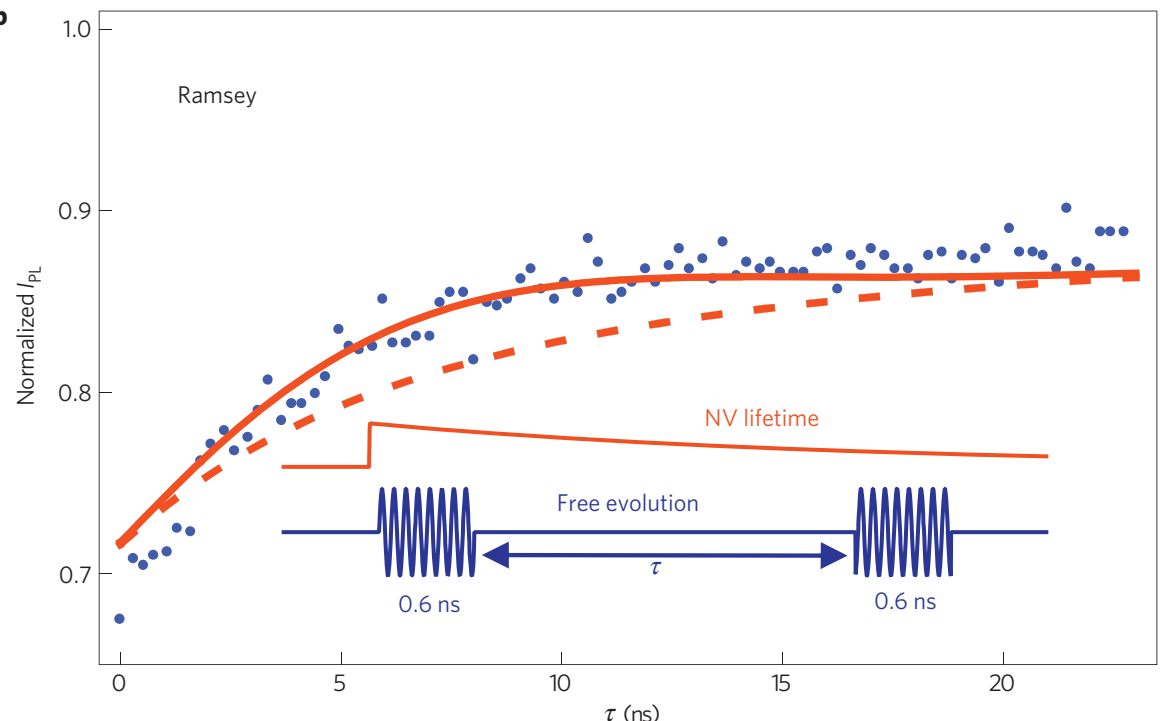

Figure 3 | Ramsey measurement of the ES hyperfine interaction. a, Bloch-sphere representation of the ES Ramsey measurement. b, ES Ramsey data, with an inset describing the pulse sequence used and the simultaneous orbital decay. The solid line is the theoretical prediction that includes the hyperfine interaction and the dashed line does not. Both predictions assume $\tau^{*}=5.8 \mathrm{~ns}$.
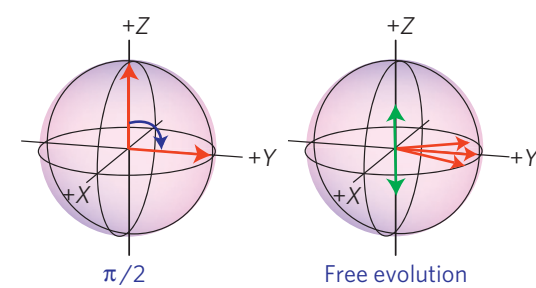

Free evolution

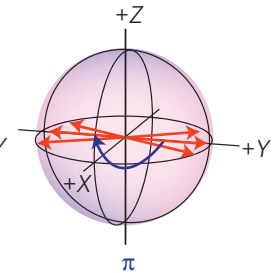

$\pi$

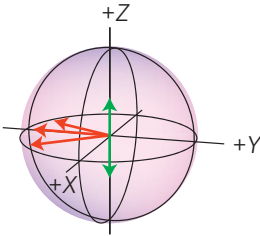

Free evolution

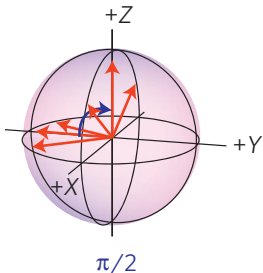

$\pi / 2$

b
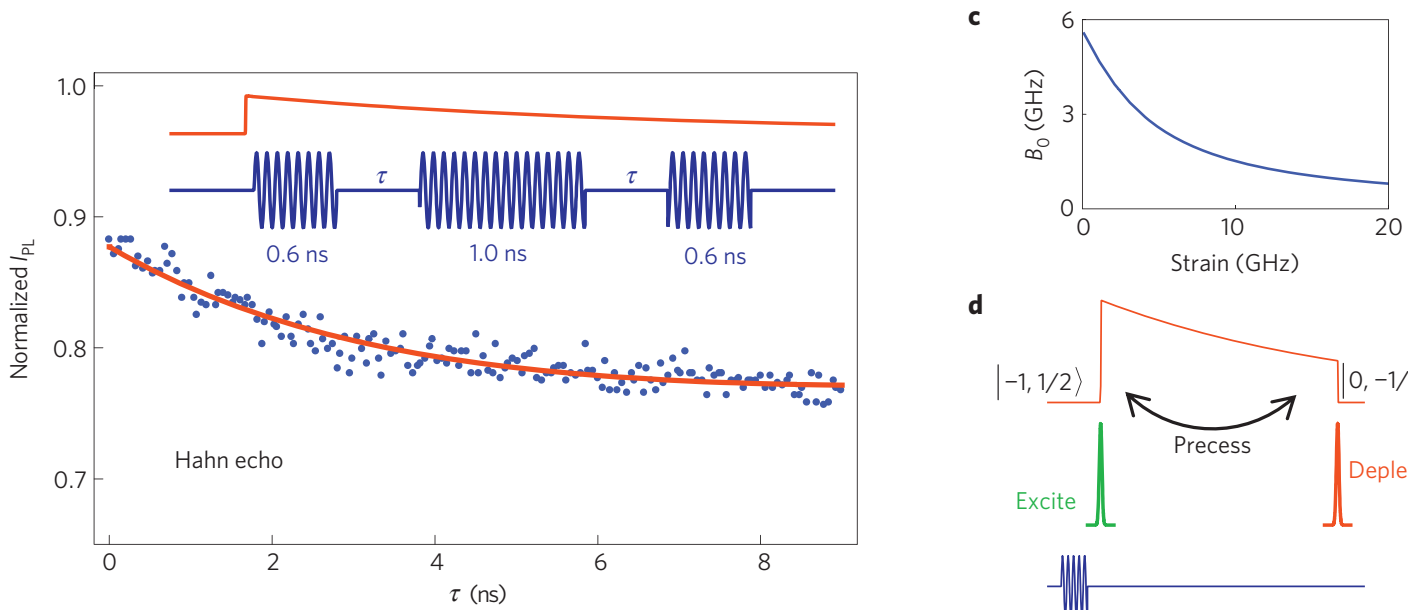

d
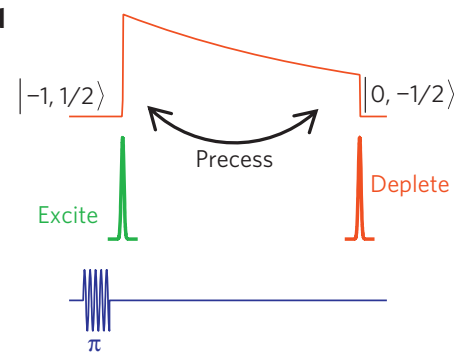

Figure 4 | ES Hahn-echo measurement of spin dephasing. a, Bloch-sphere representation of the ES Hahn-echo experiment. b, ES Hahn-echo data, with an inset showing the pulse sequence and orbital decay. The solid line is a fit to the full theory. $\mathbf{c}, B_{0}$ plotted as a function of strain. $\mathbf{d}$, Schematic of an ES swap between the $\mathrm{NV}$ centre electronic spin and the nuclear spin of nitrogen as described in the text. 
optical pulse that stimulates excitation and 'turns on' coherent precession between $|-1,+1 / 2\rangle$ and $|0,-1 / 2\rangle$ in the ES. After a $16-18$ ns precessional half-cycle, a red laser pulse stimulates emission to the ground state, swapping the state of the electronic spin into the nuclear spin.

To achieve the ES nuclear swap operation, both spin dephasing and orbital relaxation need to be controlled. Spin dephasing can be reduced by engineering higher strain to reduce $B_{0}$ (Fig. $4 \mathrm{c}$ ) and by increasing the temperature to increase $R$. Although orbital relaxation is more difficult to control, advances in fabrication may provide solutions. If the collection efficiency can be increased to near unity then we may use postselection, where the operation is repeated whenever a photon is detected before the precessional half-cycle is complete ${ }^{23}$. A number of efforts are already underway to boost collection efficiency, including the fabrication of diamond nanowires with embedded $\mathrm{NV}$ centres $^{24}$ and the development of hybrid photonic structures and waveguides ${ }^{25}$. Controlling the density of optical modes can also suppress spontaneous emission, which will minimize the number of unsuccessful attempts. Such suppression is observed in diamond nanocrystals ${ }^{26}$, and further suppression may be achieved using photonic bandgap structures tuned to overlap the energy range of the $\mathrm{NV}$ centre emission ${ }^{27}$. Although significant challenges remain, there is a clear pathway towards realizing an ES nuclear swap.

In semiconductor spintronics, spin decoherence determined by both orbital relaxation and spin dephasing is a common situation. These experiments demonstrate that fast, coherent control over an ES electronic spin can be used to differentiate between these effects and to study coherent electron-nuclear interactions. Techniques presented here may prove useful in studies of other spin systems where orbital and spin relaxation act on similar timescales. Moreover, these experimental and theoretical results provide a means to engineer the NV centre ES as a valuable quantum resource for solid-state quantum information processing at room temperature.

\section{Methods}

Samples consist of single-crystal diamond grown by chemical vapour deposition (Element-6 electronic grade). The sample described here was implanted with $10^{9}{ }^{15} \mathrm{~N}$ ions $\mathrm{cm}^{-2}$ at $20 \mathrm{keV}$ and subsequently annealed at $850 \mathrm{C}$ for $10 \mathrm{~min}$. We examine single NV centres located near the short termination of lithographically fabricated waveguides ${ }^{12}$. Photon antibunching measurements ensure that each NV centre is a single quantum emitter. We studied a total of five NV centres, which all showed consistent behaviour.

To study spin coherence during the short orbital lifetime requires on-resonance Rabi frequencies greater than $200 \mathrm{MHz}$. Such large driving fields will also stimulate transitions on nearby states within a frequency range comparable to the Rabi frequency owing to the Lorentzian pre-factor in Rabi's formula ${ }^{15}$. To constrain our manipulation to two-level dynamics and to ensure that our driving will produce spin rotation only in the excited state, we work at $B=1,276 \mathrm{G}$. At this field the ES $m_{\mathrm{s}}=0$ to $m_{\mathrm{s}}=-1$ transition frequency is $2.14 \mathrm{GHz}$. All other GS- and ES-spin transitions are strongly detuned compared with $H_{1}$. We optimized our microwave pulses at this frequency to limit the pulse rise and fall times to approximately 200 ps.

There are two sources of illumination in our experiment. The first is a $532 \mathrm{~nm}$ continuous-wave laser used for initialization and then turned off with an acousto-optic modulator. Co-aligned with this laser is fibre-coupled $554 \mathrm{~nm}$ light from an optical parametric oscillator pumped with a titanium:sapphire pulsed laser used for timed excitation. We estimate that the pulses are roughly $6 \mathrm{ps}$ in duration after passing through the fibre. They are pulse-picked to a $66 \mathrm{~ns}$ pulse period. Synchronized electronics generate microwave pulses at a fixed delay with respect to illumination.

Received 15 March 2010; accepted 28 May 2010; published online 11 July 2010

\section{References}

1. Hanson, R. \& Awschalom, D. D. Coherent manipulation of single spins in semiconductors. Nature 453, 1043-1049 (2008).
2. Jelezko, F., Gaebel, T., Popa, I., Gruber, A. \& Wrachtrup, J. Observation of coherent oscillations in a single electron spin. Phys. Rev. Lett. 92, 076401 (2004).

3. Dutt, M. V. G. et al. Quantum register based on individual electronic and nuclear spin qubits in diamond. Science 316, 1312-1316 (2007).

4. Degen, C. L. Scanning magnetic field microscope with a diamond single-spin sensor. Appl. Phys. Lett. 92, 243111 (2008).

5. Balasubramanian, G. et al. Nanoscale imaging magnetometry with diamond spins under ambient conditions. Nature 455, 648-651 (2008).

6. Maze, J. R. et al. Nanoscale magnetic sensing with an individual electronic spin in diamond. Nature 455, 644-647 (2008).

7. Fuchs, G. D. et al. Excited-state spectroscopy using single spin manipulation in diamond. Phys. Rev. Lett. 101, 117601 (2008).

8. Neumann, P. et al. Excited-state spectroscopy of single NV defects in diamond using optically detected magnetic resonance. New J. Phys. 11, 013017 (2009).

9. Rogers, L. J., McMurtrie, R. L., Armstrong, S., Sellars, M. J. \& Manson, N. B. Time-averaging within the excited state of the nitrogen-vacancy centre in diamond. New J. Phys. 11, 063007 (2009).

10. Tamarat, Ph. et al. Spin-flip and spin-conserving optical transitions of the nitrogen-vacancy centre in diamond. New J. Phys. 10, 045004 (2008).

11. Batalov, A. et al. Low temperature studies of the excited-state structure of negatively charged nitrogen-vacancy color centers in diamond. Phys. Rev. Lett. 102, 195506 (2009).

12. Fuchs, G. D., Dobrovitski, V. V., Toyli, D. M., Heremans, F. J. \& Awschalom, D. D. Gigahertz dynamics of a strongly driven single quantum spin. Science 326, 1520-1522 (2009).

13. Fu, K. C. et al. Observation of the dynamic Jahn-Teller effect in the excited states of nitrogen-vacancy centers in diamond. Phys. Rev. Lett. 103, 256404 (2009).

14. Manson, N. B., Harrison, J. P. \& Sellars, M. J. Nitrogen-vacancy center in diamond: Model of the electronic structure and associated dynamics. Phys. Rev. B 74, 104303 (2006).

15. Abragam, A. Principles of Nuclear Magnetism (Oxford Univ. Press, 1961).

16. Kubo, R. Note on the stochastic theory of resonance absorption. J. Phys. Soc. Jpn 9, 935-944 (1954).

17. van Kampen, N. Stochastic Processes in Physics and Chemistry (Elsevier, 1992).

18. Shelby, R. M., Macfarlane, R. M. \& Shoemaker, R. L. Two-pulse photon echo electron-nuclear double resonance of $\mathrm{YAlO}_{3}: \mathrm{Pr}^{3+}$. Phys. Rev. B 25, 6578-6583 (1982).

19. Jaques, V. et al. Dynamic polarization of single nuclear spins by optical pumping of nitrogen-vacancy color centers in diamond at room temperature. Phys. Rev. Lett. 102, 057403 (2009).

20. Smeltzer, B., McIntyre, J. \& Childress, L. Robust control of individual nuclear spins in diamond. Phys. Rev. A 80, 050302(R) (2009).

21. Steiner, M., Neumann, P., Beck, J., Jelezko, F. \& Wrachtrup, J. Universal enhancement of the optical readout fidelity of single electron spins at nitrogen-vacancy centers in diamond. Phys. Rev. B 81, 035205 (2010).

22. Gali, A. Identification of individual ${ }^{13} \mathrm{C}$ isotopes of nitrogen-vacancy center in diamond by combining the polarization studies of nuclear spins and first-principles calculations. Phys. Rev. B 80, 241204(R) (2009).

23. Knill, E., Laflamme, R. \& Milburn, G. J. A scheme for efficient quantum computation with linear optics. Nature 409, 46-52 (2001).

24. Babinec, T. M. et al. A diamond nanowire single-photon source. Nature Nanotech. 5, 195-199 (2010).

25. Barclay, P. E., Fu, K. C., Santori, C. \& Beausoleil, R. G. Hybrid photonic crystal cavity and waveguide for coupling to diamond NV-centers. Opt. Express. 17, 9588-9601 (2009).

26. Beveratos, A. et al. Single photon quantum cryptography. Phys Rev. Lett. 89, 187901 (2002).

27. Yablonovitch, E. Inhibited spontaneous emission in solid-state physics and electronics. Phys. Rev. Lett. 58, 2059-2062 (1987).

\section{Acknowledgements}

We gratefully acknowledge support from the AFOSR, ARO and DARPA. Work at the Ames Laboratory was supported by the Department of Energy-Basic Energy Sciences under contract No DE- AC02-07CH11358.

\section{Author contributions}

The experiment was designed and analysed by G.D.F., V.V.D., D.M.T., F.J.H., and D.D.A Measurements were made by G.D.F., D.M.T., and F.J.H. Samples were designed and fabricated by G.D.F., D.M.T., F.J.H., C.D.W., T.S., and D.D.A. All authors contributed to writing the paper.

\section{Additional information}

The authors declare no competing financial interests. Supplementary information accompanies this paper on www.nature.com/naturephysics. Reprints and permissions information is available online at http://npg.nature.com/reprintsandpermissions. Correspondence and requests for materials should be addressed to D.D.A. 